\title{
Le prix Nobel de physique 2021 attribué à deux climatologues
}

Le prix Nobel de physique 2021 a été attribué le 5 octobre dernier pour moitié à Giorgio Parisi pour ses travaux sur les systèmes complexes et pour moitié à Syukuro Manabe et Klaus Hasselmann pour leurs travaux sur la modélisation physique du climat et du changement climatique. Après le prix Nobel de chimie attribué à Paul Crutzen, Mario Molina et Sherry Rowland en 1995 et le prix Nobel de la paix attribué conjointement à $\mathrm{Al}$ Gore et au Giec en 2007, notre discipline est à nouveau à l'honneur, alors que la problématique du réchauffement climatique est plus que jamais d'actualité. Nous proposons ici quatre regards croisés de

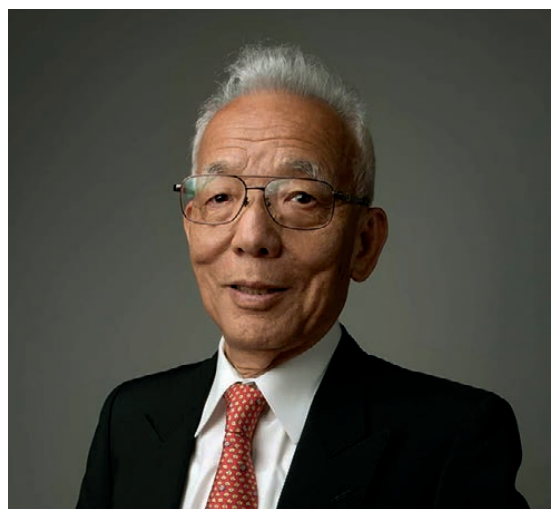

Syukuro Manabe collègues ayant côtoyé les lauréats Syukuro Manabe et Klaus Hasselmann pendant leur carrière scientifique.

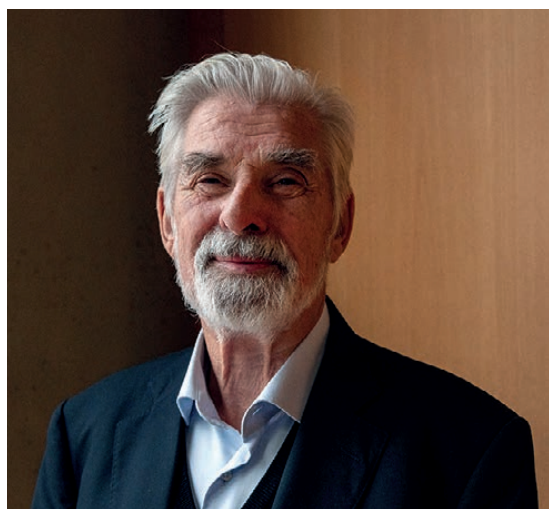

Klaus Hasselmann

\section{Syukuro Manabe, un précurseur de la modélisation du changement climatique}

Le rôle du dioxyde de carbone dans l'effet de serre remonte au XIX ${ }^{\mathrm{e}}$ siècle. Souvent attribué à Tyndall et Arrhenius, on trouve en fait la première référence à ce processus dans les travaux de la chercheuse Eunice Foote en 1857 , qui a fait cette remarque suite à ses expériences avec des bouteilles de $\mathrm{CO}_{2}$ exposées au soleil ${ }^{1}$ : «Une atmosphère de ce gaz risque de donner à la Terre une température élevée. » Il a fallu attendre plus d'un siècle pour que les travaux de Syukuro (dit Suki) Manabe, mon collègue à Princeton, permettent de vérifier cette hypothèse. Car la complexité de l'atmosphère réelle ne permet pas d'y reproduire facilement l'observation effectuée par Eunice Foote et d'autres depuis : l'excès d'énergie induit par la présence de $\mathrm{CO}_{2}$ est transporté autour de notre planète par les vents et les courants de l'océan, et sur la verticale par le processus de convection, avec plusieurs rétroactions dans le système qui peuvent amplifier ou diminuer ce réchauffement. Il n'est guère possible non plus de reproduire tout cela en laboratoire, mais le Geophysical Fluid Dynamics Laboratory (GFDL), à Princeton, où je suis arrivé trois décennies après Manabe, a développé ces expériences sur un calculateur. C'est à cette époque que la prévision numérique du temps a dépassé et fini par remplacer la prévision empirique. Manabe a été l'un des premiers à se poser la question de ce que deviendra " une prévision à l'infini ». Est-ce qu'il y a des états préférés de la circulation générale sur la planète, ce qu'on appelle des « attracteurs » dans le langage de la physique des systèmes dynamiques, et comment ces attracteurs changent-ils en réponse aux changements de conditions externes au système comme la concentration de $\mathrm{CO}_{2}$ ?

Les processus responsables de ces transports d'énergie, tels que la convection nuageuse dans l'atmosphère ou les tourbillons dans l'océan, se produisent à des échelles temporelles et spatiales largement inférieures à ce qu'on peut résoudre sur les calculateurs de l'époque des travaux pionniers de Manabe, et certaines échelles restent inaccessibles aux calculateurs d'aujourd'hui. Le génie de Manabe réside dans sa capacité d'abstraction, de faire sortir d'une situation complexe les seuls aspects nécessaires pour que le modèle ressemble au système réel qui est simulé. Reconnaissant que la convection nuageuse ne sera jamais résolue par un modèle numérique, Manabe a façonné une méthode pour extraire l'« ajustement» de l'énergie par la convection nuageuse, ce qui suffisait pour que ses simulations collent à la réalité. On dit souvent que la projection du réchauffement dû à un doublement de $\mathrm{CO}_{2}$ du premier rapport mondial sur le réchauffement de la planète, le rapport Charney de $1979^{2}$ (largement basé sur le modèle de Manabe), n'est guère moins incertaine que le dernier rapport du Giec.
Suki et moi nous sommes côtoyés souvent à Princeton pendant nos années au sein du GFDL. Les discussions entre nous portaient souvent sur l'histoire et l'avenir de la modélisation. Manabe était souvent déçu par la croissance sans fin de la complexité des modèles et leur aspect devenu aujourd'hui quasi industriel. Il voulait garder le côté ludique des modèles, permettant aux étudiant(e)s de poser des questions farfelues, de suivre leur curiosité et de s'amuser ${ }^{3}$. La leçon du prix Nobel de Manabe, ainsi que ses co-lauréats sur le podium à Stockholm, Klaus Hasselmann et Giorgio Parisi, est qu'on peut faire émerger l'essence des systèmes complexes sans en maîtriser tous les détails. Pour extraire l'ordre du désordre, la prévisibilité du chaos, il faut savoir lâcher la tentation d'exactitude en faveur de l'essentiel.

Venkatramani Balaji GFDL et Princeton University Laboratoire des sciences du climat et de l'environnement, Institut Pierre-Simon Laplace

1. https://www.climate.gov/news-features/ features/happy-200th-birthday-eunice-footehidden-climate-science-pioneer

2. https://www.nap.edu/catalog/12181/carbondioxide-and-climate-a-scientific-assessment 3. News Conference for 2021 Nobel Prize in Physics: Syukuro Manabe, 6 octobre 2021. 


\section{Suki Manabe, l'inspiration et le sourire}

Suykuro (Suki) Manabe a été un immense précurseur des modèles de climat. On pourrait écrire que sa passion, pendant des décennies, a été de comprendre la physique du climat et l'évolution du climat sous l'effet de l'augmentation du dioxyde de carbone dans l'atmosphère, en utilisant ces modèles.

Il a étudié, dès 1967, l'effet du $\mathrm{CO}_{2}$ de l'atmosphère sur la température à l'aide d'un modèle unidimensionnel, son modèle dit " radiatif convectif ». Cet article, cosigné avec Richard Wetherald, a permis de calculer le réchauffement de toute la colonne atmosphérique, en mettant en lumière l'importance de la vapeur d'eau atmosphérique, qui doublait l'effet du $\mathrm{CO}_{2}$ sur l'augmentation de la température de l'atmosphère (figure 1). Cette étude représentait un progrès considérable par rapport aux analyses scientifiques précédentes qui s'étaient intéressées à cette influence. Les résultats de cet article sont encore valables aujourd'hui.

Cette étude a ouvert la voie à toute une école de chercheurs dont le but a été d'étudier ces modèles unidimensionnels, en raffinant les processus, mais cet article, initial, contenait déjà l'essentiel des mécanismes influençant la réponse de l'atmosphère au $\mathrm{CO}_{2}$.
Manabe a ensuite développé et utilisé un modèle de circulation générale de l'atmosphère. Son modèle, en 1975, ne représentait pas toute l'atmosphère terrestre. À cause des moyens de calculs limités de l'époque, la planète modélisée avait la forme d'un " navet », car seul un tiers d'un hémisphère était décrit. Il a osé simplifier ce premier modèle, pour en extraire des résultats qu'il pouvait généraliser. Ce modèle ne représentait pas les nuages, mais l'influence essentielle de la convection était traitée. Ce modèle ne représentait pas la végétation, mais l'influence de la végétation sur l'évaporation des terres émergées était traitée. Et pourtant, avec ce bout de planète, il avait déjà trouvé un grand nombre de résultats essentiels sur le réchauffement climatique. Pourquoi les qualifier d'essentiels ? Car ils ne sont pas démentis par les modèles actuels, pourtant si sophistiqués, qu'on appelle aujourd'hui les Earth System Models. Son talent était de ne jamais rendre compte de résultats de son modèle sans s'appuyer sur une compréhension physique des mécanismes qui avaient conduit à ces résultats et sans avoir vérifié d'une façon ou d'une autre que ces mécanismes faisaient sens, grâce aux observations, si peu nombreuses par rapport à aujourd'hui, qu'il analysait.
VOL. 32, NO. 1 JOURNAL OF THE ATMOSPHERIC SCIENCES JANUARY 1975

\section{The Effects of Doubling the $\mathrm{CO}_{2}$ Concentration on the Climate of a General Circulation Model ${ }^{1}$}

\author{
Syukuro Manabe and Richard T. Wetherald \\ Geophysical Fhid Dynamics Laboratory/NOAA, Princeton Universily, Princeton, N.J. 08540 \\ (Manuscript received 6 June 1974, in revised form 8 August 1974)
}

\section{ABSTRACT}

An attempt is made to estimate the temperature changes resulting from doubling the present $\mathrm{CO}_{2}$ concentration by the use of a simplified three-dimensional general circulation model. This model contains the following simplifications: a limited computational domin, an idealized toporaph, no heat transport by ollo is computation yield some indication of how the increase of $\mathrm{CO}_{2}$ concentration may affect the distribution of temperature in the atmosphere. It is shown that the $\mathrm{CO}_{2}$ increase raises the temperature of the model troposphere, whereas it lowers that of the model stratosphere. The tropospheric warming is somewhat larger than that expected from a radiative-convective equilibrium model. In particular, the increase of surface temperature in higher latitudes is magnified due to the recession of the snow boundary and the thermal stability of the lower troposphere which limits convective heating to the lowest layer. It is also shown that the doubling of carbon dioxide significantly increases the intensity of the hydrologic cycle of the model.

Figure 1. Titre et résumé de l'article emblématique de Syukuro Manabe et Richard Wetherald sur les effets d'un doublement de la concentration atmosphérique en $\mathrm{CO}_{2}$ dans un modèle de climat tridimensionnel. L'article a été publié en 1975 dans le Journal of the Atmospheric Sciences de l'American Meteorological Society.
Quand il a couplé son modèle d'atmosphère au modèle d'océan, Manabe a encore ouvert une voie de recherches à toute une communauté. Je me souviens de bien des critiques des spécialistes de la dynamique de l'océan : ils notaient, et à juste titre, que la résolution possible, à cette époque, était insuffisante pour représenter les échanges d'énergie dans l'océan. Mais il a su, dans ce même article, présenter les faiblesses du modèle, pour ensuite insister sur les performances qui conduiraient à des progrès quand les moyens de calcul le permettraient. Aujourd'hui, les modèles couplés sont utilisés dans tous les laboratoires qui étudient le climat.

Suki Manabe avait déjà reçu de nombreux prix prestigieux, citons le Prix Revelle et le prix Crafoord, considéré comme celui qui pallie l'absence de prix Nobel en géophysique. Il est remarquable, pourtant, qu'il ait aussi reçu le prix Nobel de physique : c'est la preuve que la physique du climat, complexe, et nécessitant des moyens de calcul importants, est reconnue comme un domaine à part entière de la physique. Le fait que ses travaux aient montré l'influence des gaz à effet de serre sur le climat, qui est un problème de notre société, a aussi son importance.

Je voudrais aussi rappeler sa simplicité qui m'avait frappée lors de mes passages au GFDL, alors que je faisais mes premiers pas dans ce domaine. Il m'avait aidée à diagnostiquer l'erreur de notre premier modèle, que Robert Sadourny et moi commencions à développer au Laboratoire de météorologie dynamique ; et cela avait permis notre première publication. Je me souviens aussi de son sourire et de sa modestie lors de ce symposium en 2005, en son honneur au GFDL. Que de monde et de témoignages touchants!

J'avais eu le plaisir de le recevoir avec son épouse chez moi, lors d'un passage à Paris. Ils avaient été tous les deux des convives charmants, ouverts et enthousiastes, faisant honneur à la gastronomie française.

Katia Laval

Laboratoire de météorologie dynamique, Institut Pierre-Simon Laplace. Sorbonne Université 


\section{Klaus Hasselmann, le visionnaire}

Dès le début des années 1960, Klaus Hasselmann avait établi par des développements en série et des calculs assez complexes le rôle fondamental des interactions non linéaires entre ondes de surface dans le développement de la mer de vent et la prévision de l'état de la mer. Ces articles novateurs m'avaient fasciné, aussi ai-je été heureux qu'il m'ait invité, lors d'un séjour au Woods Hole Oceanographic Institution, à venir travailler quelque temps à Hambourg. J'y ai passé plus de deux ans, au moment où il créait l'Institut Max Planck de météorologie et développait sa théorie sur la variabilité stochastique du climat. Alors que beaucoup de chercheurs tentaient à l'époque d'expliquer les variations du climat par des influences extérieures et déterministes comme des changements de rayonnement solaire ou des rétroactions discutables, Klaus Hasselmann a montré qu'il existait une importante variabilité " naturelle » du climat, qui était due à l'impact des fluctuations du temps (vent, température, pluie) sur l'océan, la glace de mer et les autres composantes "lentes» du système climatique qui intègrent ce forçage « stochastique ». Si ce dernier n'est guère prévisible au-delà d'une dizaine de jours, les variations naturelles du climat peuvent l'être à long terme, car elles obéissent à leur propre dynamique et peuvent avoir un temps de réponse très long. Sa publication (Hasselmann, 1976) est fondamentale chercheurs de nos disciplines devraient la lire, parce qu'elle est très générale et s'applique à de nombreux aspects de la recherche climatique. Comme l'article était très théorique, faisant l'analogie avec la marche aléatoire, le mouvement brownien et d'autres concepts de physique théorique, Klaus voulait le rendre plus accessible par des applications concrètes. C'est ainsi que j'ai travaillé avec lui sur la variabilité naturelle de la température de surface océanique, qui est bien représentée - loin de l'équateur - par un modèle simple, encore utilisé de nos jours sous une forme à peine plus sophistiquée (Frankignoul et Hasselmann, 1977). En parallèle, il a lancé d'autres jeunes chercheurs sur des applications, comme la variabilité naturelle de la température globale, les variations de la couverture de glace en Arctique ou la circulation océanique profonde, suggérant toujours une approche fondamentale fondée sur des modèles aussi simples que possible, même si elle demandait des calculs approfondis. Ensuite, après avoir montré pourquoi le climat fluctue et a toujours (figure 2), si bien que tous les jeunes

\section{Stochastic climate models}

\section{Part I. Theory}

By K. HASSELMANN, Max-Planck-Institut für Meteorologie, Hamburg, FRG

(Manuscript received January 19; in final form April 5, 1976)

\section{ABSTRACT}

A stochastic model of climate variability is considered in which slow changes of climate are explained as the integral response to continuous random excitation by short period "weather" disturbances. The coupled ocean-atmosphere-cryosphere-land system is divided into a rapidly varying "weather" system (essentially the atmosphere) and a slowly responding "climate" system (the ocean, cryosphere, land vegetation, etc.). In the usual Statistical Dynamical Model (SDM) only the average transport effects of the rapidly varying weather components are parameterised in the climate system. The resultant prognostic equations are deterministic, and climate variability can normally arise only through variable external conditions. The essential feature of stochastic climate models is that the non-8veraged "weather" components are also retained. They appear formally as random forcing terms. The climste system, acting as an in. tegrator of this short-period excitation, exhibits the same random-walk response characteristics as large particles interacting with an ensemble of much smaller particles in the analogous Brownian motion problem. The model predicts "red" variance spectra, in qualitative agreement with observations. The evolution of the climate probability distribution is described by a Fokker-Planck equation, in which the effect of the random weather excitation is represented by diffusion terms. Without stabilising feedback, the model predicts a continuous increase in climate variability, in analogy with the continuous, unbounded dispersion of particles in Brownian motion (or in a homogeneous turbulent fluid). Stabilising feedback yields a statistically stationary climate probability distribution. Feedback also results in a finite degree of climate predictability, but for a stationary climate the predictability is limited to maximal skill parameters of order 0.5 .

Figure 2. Titre et résumé de l'article fondateur de Klaus Hasselmann sur l'importance des variations stochastiques du système météorologique sur le climat. fluctué, Klaus Hasselmann a développé une stratégie pour mieux détecter un signal déterministe, comme l'échauffement global, en présence de bruit, comme la variabilité naturelle du climat. C'est une méthode d'optimisation qui, à nouveau, a influencé de manière durable la recherche sur les changements climatiques. Il serait fastidieux de citer toutes ses contributions, allant de méthodes d'analyse et d'optimisation statistique à l'interprétation des observations satellitaires ou aux modèles économiques, sans compter son influence sur le développement du premier modèle de climat allemand dont il avait voulu baser, d'une manière pragmatique, la composante atmosphérique sur un modèle du Centre européen pour les prévisions météorologiques de moyen terme, mais couplé à un modèle océanique original. Ce modèle est devenu l'un des meilleurs modèles utilisés pour la prévision climatique.

Si Klaus Hasselmann a guidé et influencé de nombreux étudiants et chercheurs, il l'a fait pour contribuer à la connaissance, à la science, au progrès, le plus souvent sans même associer son nom aux travaux qu'il avait suscités. Pour moi, travailler avec lui a été une expérience stimulante qui m'a influencé tout au long de ma carrière. Son intelligence extraordinaire et sa rapidité d'esprit (et de parole) étaient parfois éblouissantes, mais pour ceux qui ont pu en profiter, c'était source d'inspiration continue. Et quelle surprise et quel émerveillement ne furent pas les nôtres lorsque, au lieu de parler de variabilité climatique comme nous l'attendions, il nous a dévoilé - était-ce lors du colloque tenu en l'honneur de ses 60 ans ? - que depuis de nombreuses années il travaillait pendant ses weekends et ses vacances sur une nouvelle théorie des champs et des particules ! Ironiquement, pour celui qui a toujours utilisé des modèles ou des concepts stochastiques, sa théorie, le modèle Metron, est un modèle déterministe. Elle vise à dériver des solutions du modèle les propriétés des particules élémentaires et les constantes universelles comme la gravité, la masse et la charge électrique. Comme si le système climatique était trop limité pour satisfaire son besoin de créer et de comprendre.

Claude Frankignoul Locean, Institut Pierre-Simon Laplace, Sorbonne Université 


\section{Klaus Hasselmann, du fondamental à l'appliqué}

Depuis les années 1970, Klaus Hasselmann a joué un rôle considérable dans le développement de la recherche sur le climat en Allemagne et en Europe. Il a non seulement créé l'Institut Max Planck de météorologie à Hambourg, mais, parce qu'il a compris l'importance des changements climatiques pour la société, il a contribué au lancement d'autres initiatives comme l'Institut pour les impacts climatiques à Potsdam (connu sous le nom de PIK), le «Climate Forum » avec la participation de nombreux représentants de l'industrie et plus récemment l'« Earth League "), qui propose des réponses aux différents problèmes globaux auxquels l'humanité est confrontée.

Mais, en même temps, Klaus Hasselmann a abordé des problèmes fondamentaux qui portent sur la dynamique du système climatique et en particulier sur les processus stochastiques qui caractérisent ce système non linéaire. Il a par exemple montré par un modèle stochastique détaillé que la longue mémoire propre à l'océan a pour effet de modifier considérablement les propriétés statistiques du système climatique dans son ensemble en favorisant les basses fréquences dans le spectre de la variabilité du système terrestre. Ces travaux l'ont conduit à emprunter à d'autres disciplines, y compris à la théorie des champs quantiques et à la physique des plasmas, des méthodologies qu'il a appliquées au système climatique. Klaus Hasselmann a toujours encouragé l'ouverture de la pensée vers des disciplines autres que celles pour lesquelles les scientifiques ont été formés. Il n'est donc pas étonnant de voir les différentes initiatives prises par Klaus Hasselmann pour appliquer certaines des approches de l'analyse stochastique à d'autres problèmes comme, par exemple, la relation qui existe entre le système climatique et le système économique.

Enfin, il faut souligner les qualités humaines d'un personnage hors du commun. Klaus Hasselmann a constitué des groupes de chercheurs qui ont joué un rôle très actif au sein de la communauté scientifique internationale. Il s'est entouré de deux directeurs à l'Institut Max Planck, Lennart Bengtsson, ancien directeur du Centre européen pour les prévisions météorologiques à moyen terme, et Hartmut Grassl, ancien directeur du Programme mondial de recherche sur le climat. Pour nous tous, ici à Hambourg, il est un véritable leader intellectuel, mais aussi un éducateur hors pair.

Guy Brasseur Max-Planck-Institut für Meteorologie

\section{Bibliographie}

Frankignoul C., Hasselmann K., 1977. Stochastic climate models, Part II: Application to sea-surface temperature anomalies and thermocline variability. Tellus, $29,289-305$. doi: 10.3402/tellusa.v29i4.11362

Hasselman K., 1976. Stochastic climate models, Part I: Theory. Tellus, 28, 473-485. doi: 10.3402/tellusa.v28i6.11316

Manabe S., Wetherald R.T., 1975. The effects of doubling the $\mathrm{CO}_{2}$ concentration on the climate of a general circulation model. J. Atmos. Sci., 32, 3-15. doi: 0.1175/1520-0469(1975)032<0003:TEODTC > 2.0.C0;2

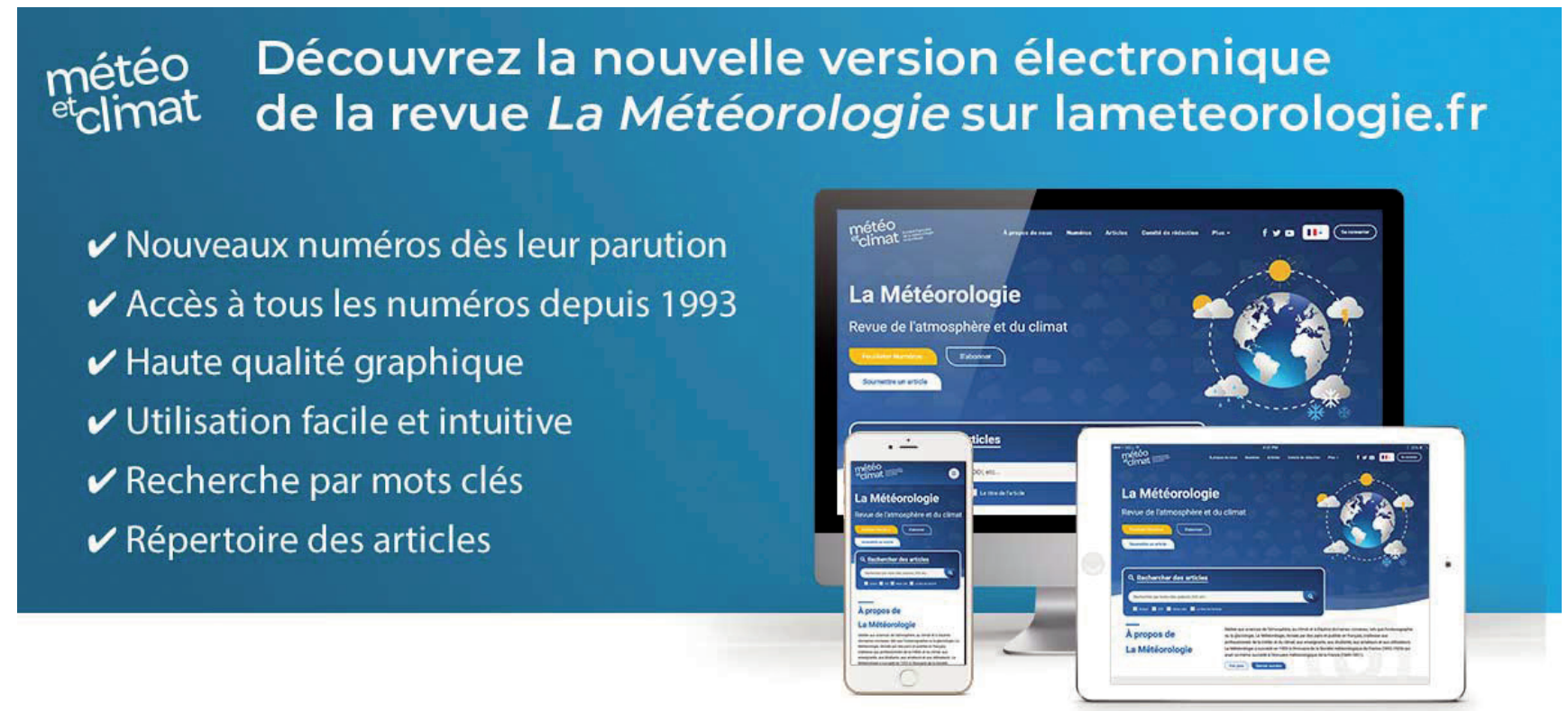

